\title{
The Impact of Bariatric Surgery on Sleep Disordered Breathing Parameters From Overnight Polysomnography and Home Sleep Apnea Test
}

\author{
Saif Mashaqi ${ }^{1}$, Kristine Steffen ${ }^{2}$, Ross Crosby ${ }^{3}$, Luis Garcia ${ }^{4}$ \\ 1. Sleep Medicine, Sanford Health 2. School of Pharmacy/pharmaceutical Sciences, North Dakota State University 3. \\ University of North Dakota, Neuropsychiatric Research Institute 4. Bariatric Surgery, University of north dakota
}

Corresponding author: Saif Mashaqi,dr_saif78@hotmail.com

\section{Abstract \\ Background}

Obstructive sleep apnea (OSA) is a common sleep disorder, especially in patients with obesity. Bariatric surgery is an effective tool to reduce weight and treat co-morbid diseases in patients with morbid obesity. One of these disorders is OSA. The most common bariatric procedures currently performed are Roux-en-Y gastric bypass (RYGB) and laparoscopic sleeve gastrectomy (LSG).

\section{Objectives}

Our study demonstrates that bariatric surgery is a very effective tool to reduce the severity of OSA, if not resolve it.

\section{Methods}

The medical charts of nine patients who had OSA and underwent bariatric surgery (LSG or RYGB) were reviewed and the apnea-hypopnea index (AHI) was compared before and after surgery. The study was conducted at the Sanford sleep center which is affiliated with the University of North Dakota School of Medicine.

\section{Results}

One patient was excluded from the statistical analysis since he was the only male patient, the remaining nine female patients had a significant reduction in AHI after surgery. The mean AHI before surgery was 40 events per hour and seven events per hour after surgery (P 0.004). The mean follow-up with sleep study after surgery was 16 months. The mean reduction in AHI was $80 \%$. There was also an improvement in oxygen saturation (SpO2) before and after surgery (90\% and 94\% respectively, P 0.008).

Received 04/11/2018 Review began 04/11/2018 Review ended 05/03/2018 Published 05/08/2018

๑) Copyright 2018

Mashaqi et al. This is an open access article distributed under the terms of the Creative Commons Attribution License CC-BY 3.0., which permits unrestricted use, distribution, and reproduction in any medium, provided the original author and source are credited.

\section{Conclusion}

The study confirms the significant reduction in AHI after bariatric surgery in female patients with OSA especially short term (one to two years postoperatively).

Categories: General Surgery, Other

Keywords: obstructive sleep apnea, bariatric surgery, roux-en-y gastric bypass, laparoscopic sleeve gastrectomy, apnea-hypopnea index, obesity

\section{Introduction}

Obesity is a very common disease in the United States (US) and worldwide. It is a challenging epidemic that is faced by the US population. The prevalence of obesity in the US in 2014 was $29 \%$ with age-adjusted prevalence being increased progressively from 23\% to 35\% from 1988 to 2012 [1]. There has been an increase in the prevalence of obesity in females compared to males over the last five to six years [2]. The most common measurement of obesity worldwide is the body mass index (BMI) which is calculated using height in meters and weight in kilograms. Based on this, the National Institutes of Health (NIH) and the World Health Organization (WHO) classified obesity into the following categories: underweight $(<18.5 \mathrm{~kg} / \mathrm{m} 2)$, normal weight (between 18.6 and $24.9 \mathrm{~kg} / \mathrm{m} 2$ ), overweight (between 25 and $29.9 \mathrm{~kg} / \mathrm{m} 2$ ), obesity class I (between 30 and $34.9 \mathrm{~kg} / \mathrm{m} 2$ ), obesity class II (between 35 and $39.9 \mathrm{~kg} / \mathrm{m} 2$ ), and morbid obesity ( $=>40 \mathrm{~kg} / \mathrm{m} 2$ ) [3].

Morbid obesity is associated with a higher risk of co-morbid diseases, including metabolic disorders (e.g., hyperlipidemia and diabetes mellitus), cardiovascular disorders (e.g., hypertension, myocardial diseases, cerebrovascular diseases, and venous thrombotic diseases), musculoskeletal disorders (e.g., osteoarthritis and gout), gastrointestinal disorders (e.g., gastroesophageal reflux disease and hepatobiliary diseases), 
genitourinary diseases, and cancer [4-6]. Sleep-related breathing disorders (e.g., OSA and sleep-related hypoventilation) are very common in patients with morbid obesity and the severity of OSA tends to be correlated with BMI [7]. One of the treatment options for morbid obesity is surgery. Weight loss surgery (i.e., bariatric surgery) is an effective method to treat obesity and decrease the risk of co-morbid conditions related to it. Bariatric surgery is indicated for BMI $>40 \mathrm{Kg} / \mathrm{m} 2$ or BMI $>35 \mathrm{~kg} / \mathrm{m} 2$ with co-morbid illnesses [8]. The most common bariatric procedures performed in the US are the Roux-en-Y gastric bypass (RYGB) and laparoscopic sleeve gastrectomy (LSG) [9]. We conducted this study to examine the impact of weight loss surgery on the apnea-hypopnea index (AHI).

\section{Materials And Methods}

We reviewed the medical charts of ten patients (one male and nine females) between the ages of 41 and 66; however, the one male patient was excluded from statistical analysis to avoid skewing of the results. All patients were morbidly obese except one (BMI $39 \mathrm{~kg} / \mathrm{m} 2$ ). We conducted overnight polysomnography (PSG) in all patients before and after bariatric surgeries (two patients underwent home sleep apnea test (HSAT) postoperatively). Seven patients underwent RYGB and three underwent LSG. The pre-operative PSGs were conducted at variable time frames from the surgery ranging from three months to six years. The postoperative PSGs and/or HSATs were conducted within 1-2 years after surgery except for one patient who had it four years after surgery. PSG parameters obtained were total sleep time, AHI, rapid eye movement (REM) AHI, periodic limb movement index (PLMI), periodic limb movement arousal index (PLMarI), spontaneous arousal index (SAI), and oxygen saturation (SpO2). The HSAT parameters obtained were (total sleep time, $\mathrm{AHI}$, and $\mathrm{SpO} 2)$.

PSGs were conducted using Embla Sandman, Version 10.1.1 (Natus Medical Inc., Pleasanton, CA, US). The obstructive AHI was calculated using the total number of obstructive apneas (defined as $=>90 \%$ reduction of the flow for $>10$ seconds) and obstructive hypopneas (using Type $1 \mathrm{~b}$ rule defined as $=>50 \%$ reduction in the flow for $>10$ seconds with associated drop in SpO2 by $=>4 \%$ ) divided by total sleep time. OSA severity was classified based on the AHI into mild $(=>5$ and $<14.9$ events per hour), moderate $\Leftrightarrow 15$ and $<29.9$ events per hour) and severe (=> 30 events per hour) [10]. HSATs were conducted using SleepMed Watermark Apnea Risk Evaluation System (ARES), model 610 (Type III) device (ARES operations, SleepMed Inc., West Palm Beach, FL, US) with ten channels. The ARES device does not stage but differentiates wake from sleep and REM from non-rapid eye movement (NREM). Arousals associated with respiratory events are scored based on a change in snoring and head movements. We used the same PSG rules to score apneas and hypopneas. The device applies a patented algorithm to calculate SpO2 and mean SpO2 (m SpO2). All PSGs and HSATs were interpreted by board-certified sleep physicians at the Sanford sleep center.

In addition to the PSG and HSAT measures, we collected the STOP-BANG score which includes loud snoring, tiredness during daytime, observed apnea, history of hypertension, BMI $>35 \mathrm{Kg} / \mathrm{m} 2$, age $>50$, neck circumference $>16$ inches, and male gender. A score of $0-2$ is less likely associated with OSA, while a score of $=>3$ is associated with high risk. The study was approved by the Sanford Health Institutional Review Board (IRB ID STUDY00001036).

\section{Results}

Starting with PSG results, four patients had severe OSA (defined as AHI $=>30$ events per hour), four patients had moderate OSA (defined as AHI $=>15$ and $<30$ events per hour) and one patient had mild OSA (defined as $\mathrm{AHI}=>5$ and $<15$ events per hour) [10]. After surgery, in the severe OSA group, one patient had the AHI normalized, one patient had AHI decreased to the moderate range, and two patients to the mild range. In the moderate OSA group, four patients had AHI normalized and one patient remained in the moderate range but dropped from 23 events per hour to 15 events per hour. In the mild OSA group, one patient had the AHI normalized (Table 1 ).

Also, we collected REM AHI which is an important PSG parameter that is affected significantly by obesity [11]. Unfortunately, five patients did not have this measure available but in the other four patients, there was a reduction in the REM AHI. Two patients progressed from the mild, moderate, and severe range to the normal range, while the other two patients moved to the mild range. SpO2 improved in all (including the male patient) but one patient (this patient's SpO2 remained the same). The improvement in SpO2 ranged between $1 \%$ and $14 \%$ (Table 2). STOP-BANG score was measured in six patients and was $=>3$ in all of them before surgery and normalized in all of them after surgery (Table 2). 


\section{Cureus}

\begin{tabular}{|c|c|c|c|c|}
\hline Variable & $\mathrm{N}$ (cases) & Before surgery (Mean +/- SD) & After surgery (Mean +/- SD) & $P$ value * \\
\hline BMI (kg/m2) & 9 & $49+/-10$ & $30.3+/-3.5$ & 0.004 \\
\hline AHI (event/hour) & 9 & $40.6+/-36.1$ & $6.9+/-7.08$ & 0.004 \\
\hline REM AHI (event/hour) & 4 & $54.5+/-12.6$ & $7.9+/-4.8$ & 0.13 \\
\hline SPO2 (\%) & 9 & $90.4+/-4.2$ & $94.2+/-1.9$ & 0.008 \\
\hline STOP-BANG *夫 & 5 & $4.8+/-1.3$ & $1+/-1$ & 0.06 \\
\hline
\end{tabular}

TABLE 1: Mean values of body mass index, polysomnograms, home sleep apnea test measures, and STOP BANG before and after bariatric surgery

* $\mathrm{P}$ value performed using Wilcoxon signed-rank test.

** STOP-BANG $=>3$ is associated with high risk of obstructive sleep apnea and $<2$ with low risk of obstructive sleep apnea.

\begin{tabular}{|c|c|c|c|c|c|c|}
\hline Case & Age & $\begin{array}{l}\text { AHI (Before } \\
\text { surgery) }\end{array}$ & $\begin{array}{l}\text { AHI (After } \\
\text { surgery) }\end{array}$ & $\begin{array}{l}\text { Follow up } \\
\text { (Months) }\end{array}$ & $\begin{array}{l}\% \text { reduction in } \\
\mathrm{AHI}\end{array}$ & $\begin{array}{l}\text { BMI after bariatric } \\
\text { surgery }\end{array}$ \\
\hline 1 & 46 & 48.4 & 2.6 & 8 & 95 & 29.5 \\
\hline 2 & 59 & 21 & 0.0 & 13 & 100 & 30 \\
\hline 3 & 64 & 10.7 & 1.2 & 15 & 89 & 33 \\
\hline 4 & 66 & 17.7 & 4 & 18 & 77 & 35 \\
\hline 5 & 49 & 117 & 21 & 10 & 82 & 33 \\
\hline 6 & 61 & 23 & 15.1 & 52 & 34 & 24 \\
\hline 7 & 54 & 82 & 8.7 & 6 & 89 & 32 \\
\hline 8 & 45 & 15.8 & 2 & 6 & 87 & 31 \\
\hline 9 & 55 & 30 & 8 & 24 & 73 & 26 \\
\hline $\begin{array}{l}\text { Mean } \\
+/ S D\end{array}$ & $\begin{array}{l}55.4+/- \\
7.6\end{array}$ & $40.6+/-36.1$ & $6.9+/-7.08$ & $16.8+/-14.4$ & $80.6+/-19.4$ & $30.4+/-3.5$ \\
\hline Median & 55.00 & 23.00 & 4.00 & 13.00 & 87.00 & 31.00 \\
\hline
\end{tabular}

TABLE 2: The apnea-hypopnea index (AHI) before and after bariatric surgery and the percent reduction in $\mathrm{AHI}$ in all patients

\section{Discussion}

Weight loss surgery is a common modality treatment that is used for rapid weight loss and subsequent control of many co-morbidities associated with morbid obesity. One of these co-morbid conditions is OSA. The success of bariatric surgery in treating sleep disordered breathing and specifically obstructive apnea has been defined variably. One definition is a postoperative reduction of $\mathrm{AHI}$ by $>50 \%$ and $\mathrm{AHI}<20$ events per hour [12]. Quintas-Neves et al. published a review article summarizing the efficacy of bariatric surgery on OSA. Out of 27 studies, nine studies focused on RYGB and only one study focused on LSG. Eight studies were prospective case series and two studies were retrospective. The sample size was around 50 patients or less in these studies except in one study by Haines et al. where they included 289 patients [13].

The follow-up time after surgery was variable in these studies (ranging between three months and five years) [14]. Some studies suggested that the greatest reduction in AHI after bariatric surgery occurs in the first year, post-op. This is followed in some cases by an increase in the AHI which is independent of BMI, suggesting other factors contributing to OSA relapse in the long-term post-op [15-16]. Other metabolic theories introduced to explain the reduction in AHI, aside from BMI reduction, include decrease in the intra- 
abdominal pressure with subsequent improvement in the oxygenation to the respiratory centers in the central nervous system [17] and the anti-inflammatory effect of weight loss and the reduction in some inflammatory biomarkers (especially TNF alpha) [18].

It is very hard to determine which bariatric surgery results in the greatest reduction in AHI since these studies had variable preoperative BMI and AHI baselines, different follow-up time, different methodology for the diagnosis of OSA (full overnight PSG vs sleep-related questionnaires), and different inclusion and exclusion criteria "as in our study". This makes it difficult to extrapolate results between these studies.

Our case series results concur with the literature results and the postop "success" definition. There was only one patient who had $<50 \%$ reduction in AHI after surgery (Figure 1). This patient had the PSG more than 4 years after surgery which suggests that this patient might have gained weight that resulted in the AHI increase, although, other metabolic factors not related to BMI can be involved. The mean follow-up time in our sample was 16 months which is within one to two years post-op that AHI is expected to drop the maximum. We also showed a reduction in REM AHI (which was not statistically significant but this could be due to a very limited sample size of just five patients) and improvement in SpO2 (Table 1).

Apnea-hypopnea index before (red) and after (green) bariatric surgery

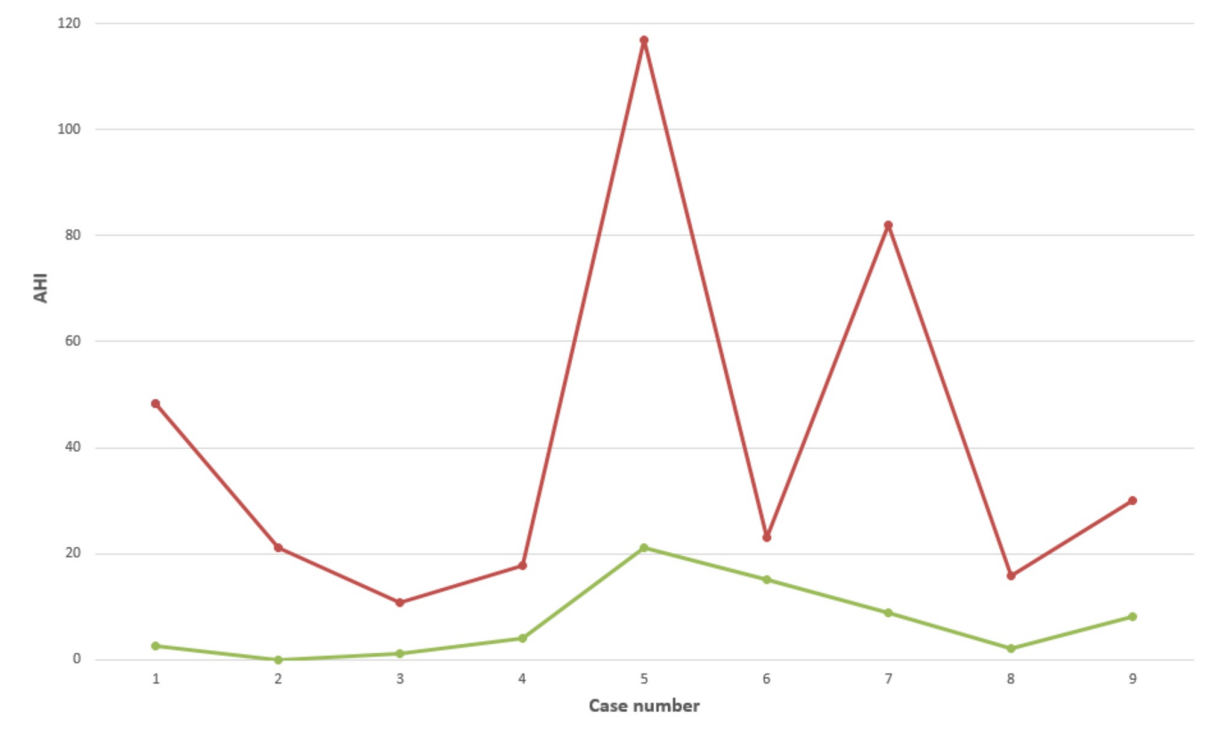

FIGURE 1: Apnea-hypopnea index before (red) and after (green) bariatric surgery

Our study had some limitations. First, it is a case series with a small sample size (nine patients). However, as noted earlier, most of the studies in the literature were case series and retrospective studies with few randomized clinical trials [16,19-20]. Second, most of our patients underwent RYGB and only three patients underwent LSG. At our bariatric and metabolic center, RYGB is the most common procedure performed. According to the American Society for Metabolic and Bariatric Surgery (ASMBS), RYGB was the most common procedure in 2012 (37\%) compared to LSG (33\%) but there has been a switch over in the last few years with LSG being the most common procedure in 2016 (58\%) compared to RYGB (19\%). Third, unfortunately, we could not obtain the whole PSG parameters pre and post-op (e.g., REM AHI) since two patients had HSAT and three patients had PSG at an outside facility which mandates cautious interpretation of these data. Fourth, all patients are females which can cause a bias in the AHI since OSA tends to be more severe in males. Finally, there were variable times at which the PSGs were obtained pre- and post-bariatric surgery.

\section{Conclusions}

Our study concurs with the body of literature that bariatric surgery (specifically RYGB and LSG) results in a significant reduction (if not normalization) in AHI at least for a short-term post-operation period (the first two years). More randomized clinical trials need to be conducted to confirm these results and explain OSA relapse that is seen in some cases in the long term, especially, if not related to weight re-gain.

\section{Additional Information}

\section{Disclosures}


Human subjects: Consent was obtained by all participants in this study. Sanford Health issued approval ID STUDY00001036. The study was approved by Sanford Health Institutional Review Board. (IRB ID STUDY00001036). Animal subjects: All authors have confirmed that this study did not involve animal subjects or tissue. Conflicts of interest: In compliance with the ICMJE uniform disclosure form, all authors declare the following: Payment/services info: All authors have declared that no financial support was received from any organization for the submitted work. Financial relationships: All authors have declared that they have no financial relationships at present or within the previous three years with any organizations that might have an interest in the submitted work. Other relationships: All authors have declared that there are no other relationships or activities that could appear to have influenced the submitted work.

\section{References}

1. Ogden CL, Carroll MD, Fryar CD, Flegal KM: Prevalence of obesity in the United States . JAMA. 2014, 312:189-90.

2. Flegal KM, Kruszon-Moran D, Carroll MD: Trends in obesity among adults in the united states, 2005 to 2014. JAMA. 2016, 315:2284-91. 10.1001/jama.2016.6458

3. National Heart, et al.: Clinical guidelines on the identification, evaluation, and treatment of overweight and obesity in adults: the evidence report. NHLBI. 1998, 6:51-209.

4. Nguyen NT, Magno CP, Lane KT, Hinojosa MW, Lane KT: Association of hypertension, diabetes, dyslipidemia, and metabolic syndrome with obesity: findings from the National Health and Nutrition Examination Survey, 1999 to 2004. J Am Coll Surg. 2008, 207:928-34. 10.1016/j.jamcollsurg.2008.08.022

5. Aune D, Sen A, Schlesinger S, et al.: Body mass index, abdominal fatness, fat mass and the risk of atrial fibrillation: a systematic review and dose-response meta-analysis of prospective studies. Eur J Epidemiol. 2017, 32:181-192. 10.1007/s10654-017-0232-4

6. Wolin KY, Carson K, Colditz GA: Obesity and cancer. Oncologist. 2010, 15:556-65. 10.1634/theoncologist.2009-0285

7. Tufik S, Santos-Silva R, Taddei JA, Bittencourt LR: Obstructive sleep apnea syndrome in the Sao Paulo Epidemiologic Sleep Study. Sleep Med. 2010, 11:441-6. 10.1016/j.sleep.2009.10.005

8. Mechanick JI, Youdim A, Jones DB, et al.: Clinical practice guidelines for the perioperative nutritional, metabolic, and nonsurgical support of the bariatric surgery patient-2013 update. Obesity. 2013, 21:1. 10.1002/oby.20461

9. Reames BN, Finks JF, Bacal D, Carlin AM, Dimick JB: Changes in bariatric surgery procedure use in Michigan, 2006-2013. JAMA. 2014, 312:959-61. 10.1001/jama.2014.7651

10. Kushida CA, Littner MR, Hirshkowitz M: Practice parameters for the use of continuous and bilevel positive airway pressure devices to treat adult patients with sleep-related breathing disorders. Sleep. 2006, 29:37580 .

11. Schwartz AR, Patil SP, Laffan AM: Obesity and obstructive sleep apnea. Pathogenic mechanisms and therapeutic approaches. Proc Am Thorac Soc. 2008, 5:185-192. 10.1513/pats.200708-137MG

12. Sher AE, Schechtman KB, Piccirillo JF: The efficacy of surgical modifications of the upper airway in adults with obstructive sleep apnea syndrome. Sleep. 1996, 19:156-77.

13. Haines KL, Nelson LG, Gonzalez R, et al.: Objective evidence that bariatric surgery improves obesity-related obstructive sleep apnea. Surgery. 2007, 141:354-8. 10.1016/j.surg.2006.08.012

14. Quintas-Neves M, Preto J, Drummond M: Assessment of bariatric surgery efficacy on obstructive sleep apnea. Rev Port Pneumol. 2016, 22:331-336. 10.1016/j.rppnen.2016.05.006

15. Pillar G, Peled R, Lavie P: Recurrence of sleep apnea without concomitant weight increase 7.5 years after weight reduction surgery. Chest. 1994, 106:1702-4.

16. Feigel-Guiller B, Drui D, Dimet J, et al.: Laparoscopic gastric banding in obese patients with sleep apnea: a 3-year controlled study and follow-up after 10 years. Obes Surg. 2015, 23:1886-92. 10.1007/s11695-0151627-5

17. Sarkhosh K, Switzer NJ, El-Hadi M, et al.: The impact of bariatric surgery on obstructive sleep apnea: a systematic review. Obes Surg. 2013, 23:414-423. 10.1007/s11695-012-0862-2

18. Pallayova M, Steele KE, Magnuson TH, et al.: Sleep apnea determines soluble TNF- $\alpha$ receptor 2 response to massive weight loss. Obes Surg. 2011, 21:1413-23. 10.1007/s11695-011-0359-4

19. Dixon JB, Schachter LM, O'Brien PE, et al.: Surgical vs conventional therapy for weight loss treatment of obstructive sleep apnea: a randomized controlled trial. JAMA. 2012, 308:1142-9. 10.1001/2012.jama.11580

20. Aguiar IC, Freitas WR, Santos IR, et al.: Obstructive sleep apnea and pulmonary function in patients with severe obesity before and after bariatric surgery: a randomized clinical trial. Multidiscip Respir Med. 2014, 9:43. 10.1186/2049-6958-9-43 